\title{
Author Correction: Pathogenic stromal cells as therapeutic targets in joint inflammation
}

Stephanie G. Dakin (D), Mark Coles, Jonathan P. Sherlock, Fiona Powrie, Andrew J. Carr and Christopher D. Buckley (D)

Nature Reviews Rheumatology (2018) https://doi.org/10.1038/s41584-018-0112-7

Published online 12 November 2018

In the originally published version of this article, the acknowledgements and affiliations contained errors. In the acknowledgements, "NIHR Oxford and Birmingham Biomedical Research Centres" and "the Department of Health and Social Care" were incorrectly presented as "NIHR Oxford Biomedical Research Centres" and "the Department of Health". For affiliation 4, "NIHR Birmingham Biomedical Research Centre, University Hospitals Birmingham NHS Foundation Trust and University of Birmingham, Institute of Inflammation and Ageing, Birmingham, UK" was incorrectly presented as "Institute of Inflammation and Ageing, University of Birmingham, Birmingham, UK". For affiliation 3, "Institute" was incorrectly spelt. These errors have now been corrected in the HTML and PDF versions of the manuscript.

https://doi.org/10.1038/s41584-018-0134-1 I Published online 29 November 2018

\section{Author Correction: Decoding osteoarthritis}

\section{Michelle Trenkmann}

Nature Reviews Rheumatology (2018) https://doi.org/10.1038/s41584-018-0131-4 Published online 28 November 2018

In the online version of this article, there was an error in the text. The line beginning "A majority of the identified common variants with $>5 \%$ frequency (variants with a minor allele) [...]" has been corrected to "A majority of the identified common variants (variants with a minor allele frequency $>5 \%$ ) [...]". This error has now been corrected in the HTML and PDF versions of the manuscript.

https://doi.org/10.1038/s41584-018-0140-3 I Published online 7 December 2018 